\title{
Pathologische Frakturen bei malignen Knochentumoren
}

\author{
Markus Schultheiss, Lothar Kinzl, Alexandra von Baer, \\ Erich Hartwig, Markus Arand
}

\section{Zusammenfassung}

Maligne Knochentumoren sind im Rahmen von multimodalen Behandlungskonzepten zu therapieren. Gerade im Fall einer initial oder während der Behandlung auftretenden pathologischen Fraktur muss die Therapie dieser Tumoren in geeigneten Tumorzentren erfolgen. Im Rahmen dieser Arbeit werden die aktuellen interdisziplinären Therapiekonzepte unter Berücksichtigung der pathologischen Fraktur erörtert.

\section{Pathological Fractures due to Malignant Bone Tumors}

Malignant bone tumors should be treated within interdisciplinary therapy concepts. Especially when pathological fractures occur at the beginning of or during therapy, the patient has to be treated in a specialized center. This article summarizes actual interdisciplinary treatment concepts with special consideration of pathological fractures.

\section{Einleitung}

Die Behandlung maligner Knochentumoren fordert die interdisziplinäre Zusammenarbeit heraus [1-6].

Kontrollierte onkologische Therapiekonzepte zum einen sowie optimierte Bildgebung im MRT und PET-CT, welche die Tumorausbreitung lokal wie auch die Metastasierung genauer und früher erkennen lässt, sind wesentliche Voraussetzungen von Therapieverbesserungen.

Das Osteosarkom ist mit einem Anteil von 35-48\% der häufigste maligne Knochentumor, gefolgt von dem Chondrosarkom mit 20-26\% und dem Ewing-Sarkom mit 10-16\%: Das Osteo- und das Ewing-Sarkom gehören zu den malignen Tumoren, die bevorzugt im Kindes- und Jugendalter vorkommen, während das Chondrosarkom gehäuft in der sechsten Lebensdekade auftritt [7].

Osteosarkome und Ewing-Tumoren sind hochmaligne Tumoren, die durch rasche und frühe Metastasierung gekennzeichnet sind. Zum Zeitpunkt der Diagnose- stellung sind Metastasen zwar erst bei 10-20\% der Betroffenen bereits klinisch verifizierbar, obwohl davon auszugehen ist, dass in $80 \%$ bereits eine Mikrometastasierung vorliegt.

Die Prognose maligner Knochentumoren hat sich in den letzten zwei Jahrzehnten entscheidend verbessert. Aufgrund der neoadjuvanten Chemotherapie bei Osteo- und Ewing-Sarkomen konnte die Überlebensrate von nur 10-20\% auf $>60 \%$ gesteigert werden. Dabei bleibt die operative Tumorentfernung entscheidender Bestandteil des multimodalen Therapieansatzes.

Schmerzen sind das führende Leitsymptom, sie entwickeln sich neben einer lokalen Schwellung über einen Zeitraum von mehreren Wochen bis Monaten, werden als tief und bohrend beschrieben und zumeist verharmlost.

In $5-10 \%$ der Patienten wird die Erkrankung aufgrund einer pathologischen Fraktur diagnostiziert [5, 9].

\section{Hauptteil \\ Diagnostik}

Die Röntgenuntersuchung ist zur Beurteilung von Dignität und Art eines Knochentumors aufgrund der verschiedenen Destruktionsmuster von größter Bedeutung: Bei Malignitätsverdacht ist die MRT die Methode der Wahl für eine weiterführende Abklärung. Tumorgrenzen und damit das Resektionsausmaß lassen sich wesentlich genauer differenzieren. Besonders die intramedulläre Tumorausdehnung und die Weichteilbeteiligung lassen sich in multiplanaren Schnittbildverfahren besonders gut darstellen. Es können sowohl Skipmetastasen (,Satellitenknoten“) verifiziert werden als auch die Infiltration von Gefäß-Nerven-Bündeln und der Muskelkompartimente. Die Computertomographie ist gegebenenfalls ergänzend notwendig, um ossäre Strukturen besser beurteilen zu können; sie sind zur Diagnostik von etwaigen Lungenmetastasen unerlässlich. Neuerdings wird im Rahmen des präoperativen Stagings und zur Verlaufskontrolle das PET(Positronen-Emissions-Tomogramm) -CT als dynamische Untersuchung zur Beurteilung der Tumorvitalität und der Fernmetastasierung genutzt.

\section{Biopsie}

Die Diagnose eines bösartigen Knochentumors wird immer aufgrund der feingeweblichen Untersuchung des im Rahmen einer Probebiopsie gewonnenen repräsentativen Tumorgewebes gestellt.

Das chirurgische Vorgehen bei der Probebiopsie von Knochentumoren ist eine verantwortungsvolle Aufgabe und sollte deshalb möglichst in der Klinik durchgeführt werden, in der auch die eigentliche Extremitäten erhaltende Tumorresektion erfolgt. Ansonsten sind Diagnosefehler und Komplikationen bis zu zwölfmal häufiger, wenn nicht sogar eine Ex- 
tremitäten erhaltende Tumorresektion unmöglich.

Der Zugang zur Biopsie hat immer im Verlauf der späteren Hautinzision zu liegen und wird bei der späteren Operation spindelförmig exzidiert. Drainagen sollten immer distal unmittelbar am Wundpol ausgeleitet werden, um den Drainagenkanal bei der endgültigen Resektion ebenfalls mitzuentfernen.

Die Beurteilung der Gewebspathologie erfordert die Kenntnis der exakten Lokalisation sowie der grundsätzlichen radiologischen Veränderungen. Im Rahmen der Behandlung an einem Zentrum sollte das Bildmaterial deshalb auch dem Pathologen vorliegen.

Die Stadieneinteilung maligner Läsionen des Bewegungsapparates (Tab.1) [11] erfolgt ebenso wie die der Resektionsgrenzen muskuloskelettaler Tumoren nach Enneking (Tab.2). Zur Graduierung des Ansprechens einer neoadjuvanten Chemotherapie wird im deutschsprachigen Raum die Einteilung nach SalzerKuntschik herangezogen (Tab.3) [10]. Regressionsgrade I bis III sind prognostisch günstiger als solche mit Regressionsgraden IV bis VI. Patienten mit den Regressionsgraden IV bis VI werden als „Non-Responder“ bezeichnet.

Auch die Einschätzung der mechanischen Stabilität des betroffenen Knochens erfolgt konventionell, ggf. unter Zuhilfenahme der Computertomographie (CT). Hilfreich hierfür ist der Score nach Mirels der zur Abschätzung des Frakturrisikos bei Metastasierung herangezogen wird (Tab.4) [8]. Jedem der genannten 4 Parameter werden 3 Graduierungen zugeordnet, die Summation ergibt Punktezahl zwischen 4 und 12. Das Risiko einer Fraktur bis zu einer Punktezahl von 7 liegt unter $5 \%$, bei einer Punktezahl von 8 steigt das Risiko auf $15 \%$. Ab einer Punktezahl von 9 liegt die Frakturwahrscheinlichkeit über $33 \%$, eine palliative Stabilisierung wird vorgeschlagen.

\section{Studien}

Maligne Knochentumoren müssen heute nach international anerkannten Studienprotokollen behandelt werden.

Folgende Therapiestrategien (Tab.5) sind im Rahmen der Nomenklatur zu unterscheiden $[1-4,6]$ :

Tab.1 Stadieneinteilung maligner Läsionen des Bewegungsapparates nach Enneking

\begin{tabular}{lllllll} 
& IA & IB & IIA & IIB & IIIA & IIIB \\
\hline Grade & G1 & G1 & G2 & G2 & G1-2 & G1-2 \\
Ausdehnung & T1 & T2 & T1 & T2 & T1 & T2 \\
Metastasen & M0 & M0 & M0 & M0 & M1 & M1
\end{tabular}

Tab.2 Einteilung der Resektionsgrenzen muskuloskelettaler Tumoren nach Enneking

\begin{tabular}{lll} 
Resektionsebene & Pathologisches Ergebnis & \\
\hline Intrakapsulär & Intraläsional & Resektionsrand im Tumor \\
\hline Marginal & $\begin{array}{l}\text { Extrakapsulär, aber im beglei- } \\
\text { tenden reaktiven Gewebe }\end{array}$ & $\begin{array}{l}\text { Reaktives Gewebe eventuell mit } \\
\text { Satellitenläsionen des Tumors }\end{array}$ \\
\hline Weit & $\begin{array}{l}\text { Außerhalb des reaktiven, im } \\
\text { normalen Gewebe }(2-3 \mathrm{~cm})\end{array}$ & Tumorfreier Resektionsrand \\
\hline Radikal & Extrakompartimental & Tumorfreier Resektionsrand
\end{tabular}

Tab.3 Regressionsgrade nach Salzer/ Kuntschnik für maligne Knochentumoren

\begin{tabular}{ll} 
Grade & Effekte \\
\hline I & Keine vitalen Tumorzellen \\
\hline II & $\begin{array}{l}\text { vereinzelt nachweisbare } \\
\text { Tumorzellen oder eine vitale } \\
\text { Tumorinsel von }<0,5 \mathrm{~cm}\end{array}$ \\
\hline III & $<10 \%$ vitales Tumorgewebe \\
\hline IV & $10-50 \%$ vitales Tumorgewebe \\
\hline V & $>50 \%$ vitales Tumorgewebe \\
\hline VI & $\begin{array}{l}\text { kein Effekt der Chemotherapie } \\
\text { erkennbar }\end{array}$
\end{tabular}

Vorteile einer neoadjuvanten Chemotherapie bestehen darin, die überlebenswichtige systemische Therapie ohne Zeitverzögerung zu beginnen. Diese ermöglicht oftmals erst durch ein „Downstaging“, d.h. eine chemotherapeutisch induzierte Tumorschrumpfung die Extremitäten erhaltende Operation. Nach einer neoadjuvanten präoperativen Chemotherapie ergibt sich die Möglichkeit, die Wirksamkeit der eingesetzten Medikamente histologisch zu evaluieren und zu klassifizieren, um die postoperative Therapie je nach Regressionsgrad zu planen.

Tab.4 Risiko für eine pathologische Fraktur der langen Röhrenknochen nach Mirels

\begin{tabular}{llll}
\hline Punktwert & $\mathbf{1}$ & $\mathbf{2}$ & $\mathbf{3}$ \\
\hline Lokalisation & Obere Extremität & Untere Extremität & Peritrochantär \\
\hline Schmerz & Gering & Mäßig & Stark \\
\hline Struktur & $\begin{array}{l}\text { Osteoplastische } \\
\text { Metastase }\end{array}$ & $\begin{array}{l}\text { Gemischte } \\
\text { Metastase }\end{array}$ & $\begin{array}{l}\text { Osteolytische } \\
\text { Metastase }\end{array}$ \\
\hline Ausdehnung & $\begin{array}{l}\text { Unter } 1 / 3 \text { des Kno- } \\
\text { chendurchmessers }\end{array}$ & $\begin{array}{l}1 / 32^{2} / 3 \text { des Knochen- } \\
\text { durchmessers }\end{array}$ & $\begin{array}{l}\text { über } 2 / 3 \text { des Kno- } \\
\text { chendurchmessers }\end{array}$
\end{tabular}

Tab.5 Varianten der systemischen Chemotherapie

Varianten Präoperative plus postopera1. tive Chemotherapie (= neoadjuvante) bei kurativem multimodalen Konzept (Studien)

2. Postoperative Chemotherapie (= adjuvante) bei multimodalem Konzept mit kurativer Intention

(Studien)

3. Palliative Chemotherapie bei metastasierten Sarkomen (Standard + Studien)
Im Folgenden wird näher auf die zuvor genannten malignen Knochentumoren eingegangen und die operative Therapie dargestellt.

\section{Osteosarkom}

Das Osteosarkom ist ein hochmaligner, primärer Knochentumor. Histologisch unterscheidet man 5 verschiedene Subtypen $[1-3,5,6]$ :

- klassisch

- teleangiektatisch

- periosteal

- parossal

- zentral, niedrig maligne 
Die Erkrankung tritt gehäuft im zweiten Lebensjahrzehnt (60\%) auf. Ungefähr $30 \%$ der Erkrankten sind älter als 40 Jahre. Lange Röhrenknochen sind am häufigsten betroffen (in 60\% distales Femur, proximale Tibia oder proximaler Humerus). Die Lokalisation ist in $>90 \%$ metaphysär und in nur $9 \%$ diaphysär.

Im Rahmen der im deutschsprachigen Raum durchgeführten COSS-Studien (COSS: Cooperative Osteosarkom Studie) kann aufgrund der Erfahrungen aus diesen bislang neoadjuvanten Chemotherapieprotokollen im Rahmen eines interdisziplinären Therapiekonzeptes ( T4/T5 Studie 1975, COSS 1977, COSS 1986 und COSS 1996) derzeit eine 5-Jahres-Überlebensrate von $60-70 \%$ erreicht werden. Der Prozentsatz Extremitäten erhaltender Operationen liegt inzwischen über $80 \%$.

Seit 2004 wird eine Kooperationsstudie verschiedener internationaler Studien (Amerika, Skandinavien, Europa) durchgeführt. Diese EURAMOS ( European and American Osteosarcoma Study Group gegründet 2002) -Studie ist in Tab.6a u. b näher spezifiziert.

Die sich der neoadjuvanten Chemotherapie anschließende Operation muss unter onkologisch radikalen Gesichtspunkten erfolgen, eine weite bzw. radikale Resektion ist anzustreben. In Abhängigkeit vom histologisch verifizierten Tumoransprechen auf die Chemotherapie wird die postoperative Chemotherapie geplant.

Seit 2004 gibt es speziell für Patienten >41-65 Jahre das EURO B.O.S.S. I Therapieschema, das in Tabelle 6 näher spezifiziert ist. Es wurde aufgrund der schlechteren Heilungsrate in dieser Altersgruppe eine adjuvate oder neoadjuvante Behandlung initiiert. Im Rahmen der adjuvanten Therapiegruppe ist auch die primäre Operation möglich.

Prognostische Faktoren in der Behandlung von Osteosarkomen sind bereits bei Diagnosestellung erkennbar. Tumorgröße (>100 ml Volumen), Tumorlokalisation am Rumpf und das Vorhandensein von synchronen pulmonalen Metastasen sind schwieriger, jedoch unter kurativem Ansatz therapierbar. Die unvollständige Tumoroperation, ein Lokalrezidiv und die metachrone Metastasierung sind prognostisch als überaus schlecht einzustufen.

Tab.6a EURAMOS Therapieschema

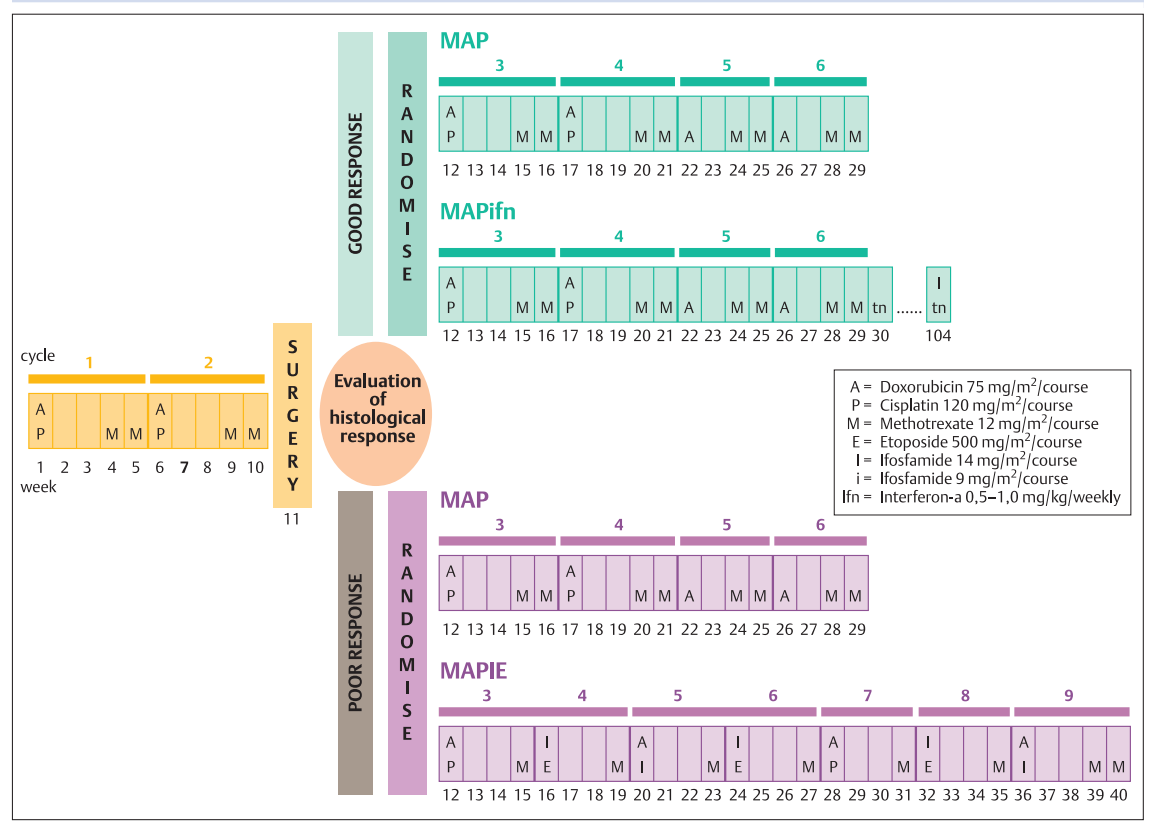

Tab.6b EURO B.O.S.S. I Therapieschema

Patienten mit primärer Operation: adjuvante Chemotherapie

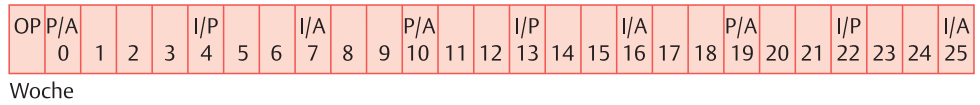

Gutes und intermediäres Ansprechen (<50\% vitale Tumorzellen)

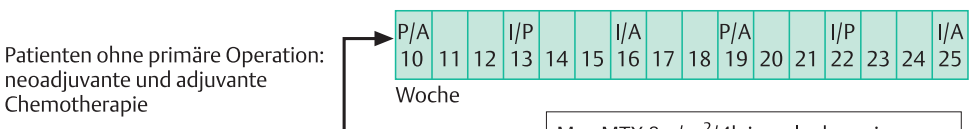

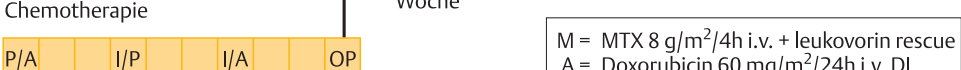

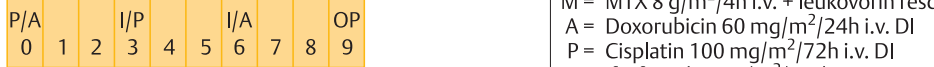

$$
\begin{aligned}
& \begin{array}{llllllll} 
& & &
\end{array} \\
& \text { Sehr schlechtes Ansprechen ( } \geq 50 \% \text { vitale Tumorzellen) } \\
& \begin{array}{|l|l|l|l|l|l|l|l|l|l|l|l|l|l|l|l|}
\hline \text { P/A } & \text { M I/P } & \text { M I/A } & & \text { M P/A } \\
\hline
\end{array} \\
& \begin{array}{lllllllllll|l|l|l|l|l|l|l|}
10 & 11 & 12 & 13 & 14 & 15 & 16 & 17 & 18 & 19 & 20 & 21 & 22 & 23 & 24 & 25 \\
\hline
\end{array} \\
& \text { Woche } \\
& \begin{array}{|l|l|l|l|l|}
\hline \text { M } & \text { I/P } & & \text { M } & \text { I/A } \\
\hline
\end{array} \\
& \begin{array}{l|l|l|l|l|}
26 & 27 & 28 & 29 & 30
\end{array} \\
& \text { Woche }
\end{aligned}
$$

\section{Ewing-Sarkom}

Das „Ewing-Sarkom“ gehört mit dem malignen peripheren neuroektodermalen Tumor (MPNT oder PNET) zur Familie der Ewing-Tumoren. Gemeinsam sind sie durch eine ews/ets-Translokationen charakterisiert. Ewing-Tumoren treten im Alter zwischen 3 und 30 Jahren auf [4].

Zytogenetisch kann bei $80 \%$ aller histologisch klassifizierten Ewing-Tumoren eine Veränderung am Chromosom 22 gefunden werden (22q12). Der Nachweis einer $\mathrm{t}(11 ; 22)(\mathrm{q} 24 ; \mathrm{q} 12)$ oder einer $\mathrm{t}(21 ; 22)$ (q22;q12) ist pathognomonisch für die
Diagnose der Ewing-Tumoren. Die häufigste Lokalisation ist das Becken, gefolgt von Femur und Rippen.

Im Gegensatz zum Osteosarkom, bei dem die Metaphysen der langen Röhrenknochen die bevorzugte Lokalisation darstellt, ist der Ewing-Tumor typischerweise an der Diaphyse lokalisiert. Die Metastasierung betrifft primär die Lunge, aber auch das Skelettsystem oder das Knochenmark.

Das Ewing-Sarkom ist eine systemische Erkrankung und erfordert daher eine multimodale, systemische Therapie. Stu- 

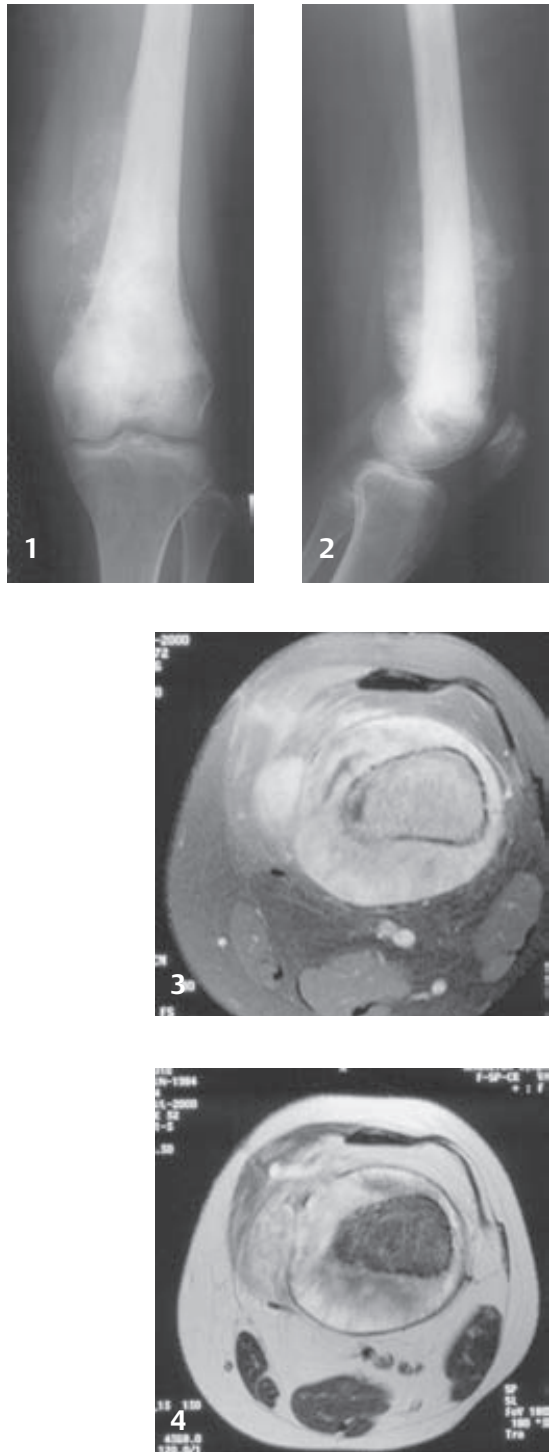

dien (CESS 81, CESS 86, EICESS 92) haben gezeigt, dass die Kombination von Chemotherapie, Resektion und Strahlentherapie einer alleinigen Resektion überlegen ist. Das aktuelle Protokoll (EUROE.W.I.N.G. 99) ist in Tabelle 7 näher spezifiziert.

Neben dem Tumorvolumen ( $<200$ vs. $>200 \mathrm{ml}$ ) hat auch der Response auf die präoperative Chemotherapie ( $<10$ vs. $>10 \%$ vitaler Tumoranteil im Resektat) einen wesentlichen Einfluss auf die Prognose. Hat der Tumor auf die neoadjuvante Chemotherapie schlecht angesprochen ( $>10 \%$ vitaler Tumor) oder ist das initiale Tumorvolumen hoch (>200 ml), so wird von verschiedenen Gruppen oft eine Nachbestrahlung gefordert.

Etwa $30-40 \%$ der Patienten mit einem Ewing-Tumor erleiden ein Rezidiv. Patienten mit einem frühen Tumorrezidiv
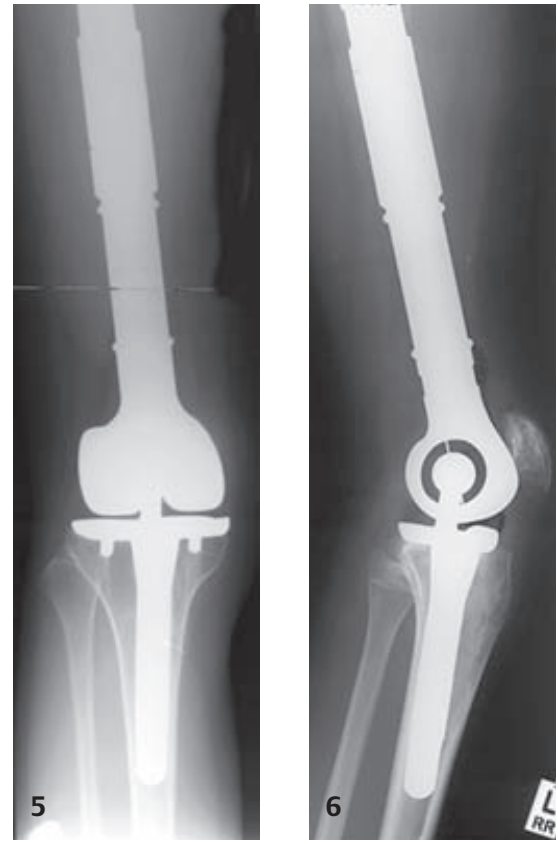

Abb.1-6 (siehe Tab. 9) male Chondrosarkom zu erkennen. Denn dieses ist chemo- und strahlentherapiesensibel. Deshalb wird hierbei dasselbe therapeutische Vorgehen wie für die Ewing- oder Weichteilsarkome empfohlen.

Das Chondrosarkom stellt somit eine besondere Herausforderung an die operative Behandlung, denn die Prognose wird durch eine optimale weite bzw. radikale Resektionstechnik bestimmt. Da nach Operationen häufig Rezidive auftreten, sind primär bei G1-/G2-Tumoren auch große Eingriffe gerechtfertigt. In einem Lokalrezidiv zeigt sich zudem oft eine Progression zu dedifferenzierten Tumoren (Dedifferenzierung niedrigmaligner Chondrosarkome).

Das intraläsionale Prozedere wird deshalb für das Chondrosarkom auch bei niedrigem Malignitätsgrad abgelehnt. Bei adäquater Resektion wird eine Heilung in $60 \%$ erreicht, wenn keine simultanen Metastasen bestehen. Pulmonale Metastasen werden wenn möglich ggf. chirurgisch entfernt.

\section{Operative Verfahren}

Die verschiedenen zur Verfügung stehenden Operationstechniken werden in drei Gruppen zusammengefasst:

- Ablative Maßnahmen wie Amputation oder Exartikulation.

- Resektions-Rekonstruktions-Verfahren mit Defektersatz durch modulare Endoprothesen oder Allo- bzw. Autograft (klinisches Fallbeispiel siehe Abb. 1-6).

( $<2$ Jahre nach Diagnosestellung) weisen eine deutlich schlechtere Prognose auf (5-Jahres-Überleben: 5\%) als Patienten mit einem späten Rezidiv (5-Jahres-Überleben: 35\%). Eine nochmalige aggressive Chemotherapie in Verbindung mit Tumorresektion oder Strahlentherapie verbessert die Prognose erneut signifikant.

\section{Chondrosarkom}

$10 \%$ aller malignen Knochentumoren sind Chondrosarkome, welche gehäuft zwischen dem 4. und 6. Lebensjahrzehnt auftreten. Das Becken ist in $70 \%$ der Fälle die bevorzugte Lokalisation [7].

Bisher gibt es kein adäquates Chemotherapieschema und auch mit Strahlentherapie liegen nur begrenzt positive Erfahrungen in der Behandlung von Chondrosarkomen vor. Wichtig ist es jedoch als histologische Sonderform das mesenchy-
- Resektions-Replantations-Verfahren wie die Umkehrplastiken (klinisches Fallbeispiel siehe Abb.7).

Zur Defektüberbrückung stehen derzeit folgende rekonstruktive Verfahren zur Verfügung:

- Autogene, gefäßgestielte Fibula

- Allogenes Diaphysensegment

- Kallusdistraktion

- Modulare Tumor-Spezialprothesen und Custom made-Prothesen

(Abb.1-6)

- Umkehrplastik, z.B. Borggreve

(Abb. 7).

\section{Pathologische Frakturen}

Pathologische Frakturen sind bei niedrigmalignen Knochentumoren selten, treten jedoch in $10 \%$ bei hochmalignen Knochentumoren auf (Abb.8-9) [5, 9]. 


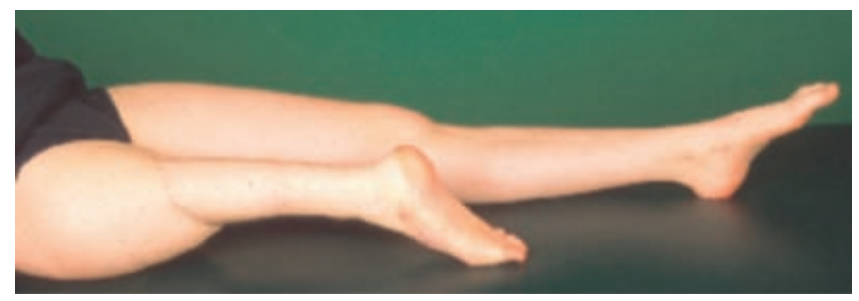

Abb. 7 Klinisches Beispiel für eine Borggreve Umkehrplastik.

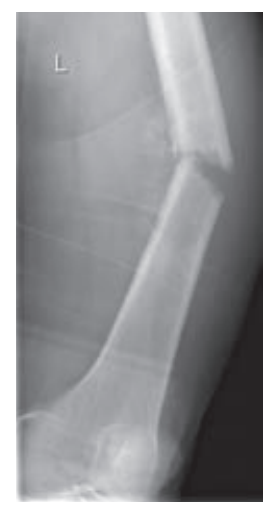

Abb. 8 Pathologische Frakturierung eines Osteosarkoms am Oberschenkel 12/06 (konventionelles Röntgen).

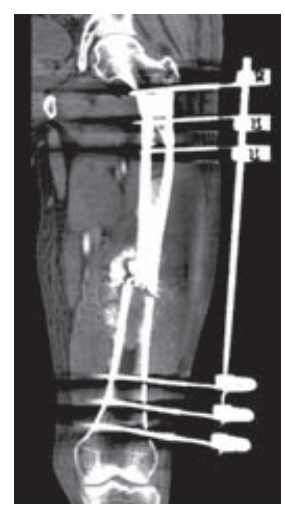

Abb. 9 Externe Stabilisierung nach pathologischer Fraktur bei Osteosarkom vor neoadjuvanter Chemotherapie $1 / 06$ (CT Scan).

Beim Auftreten einer pathologischen Fraktur ist die Prognose insgesamt eher schlechter, da die Fraktur für ein großes Tumorvolumen und eine besonders hohe Aggressivität spricht. Auch im Falle einer pathologischen Fraktur muss das wichtigste Ziel sein, die Patienten gemäß den oben angeführten multimodalen Therapiekonzepten zu behandeln. Dazu sind in Anlehnung an Mutschler folgende Regeln einzuhalten (Tab.8) [9]:

Ein klinisches Fallbeispiel ist in Abb. 1014 illustriert.

\section{Schlussfolgerung}

Besonders im Fall der pathologischen Fraktur von malignen Knochentumoren sind die therapeutischen Überlegungen immer im Rahmen eines interdisziplinären, onkologische Knochenexpertenteams zu diskutieren.

Die Behandlung sollte ausschließlich interdisziplinär an einem Zentrum erfol gen und wenn möglich im Rahmen derzeit aktueller Studienprotokolle oder in Anlehnung an diese.

Nach Einschätzung der Gesamtsituation und der Gesamtprognose sind intermittierend auch individuelle Lösungen ab-
Tab. 7 EURO-E.W.I.N.G. 99 Therapieschema für das Osteosarkom

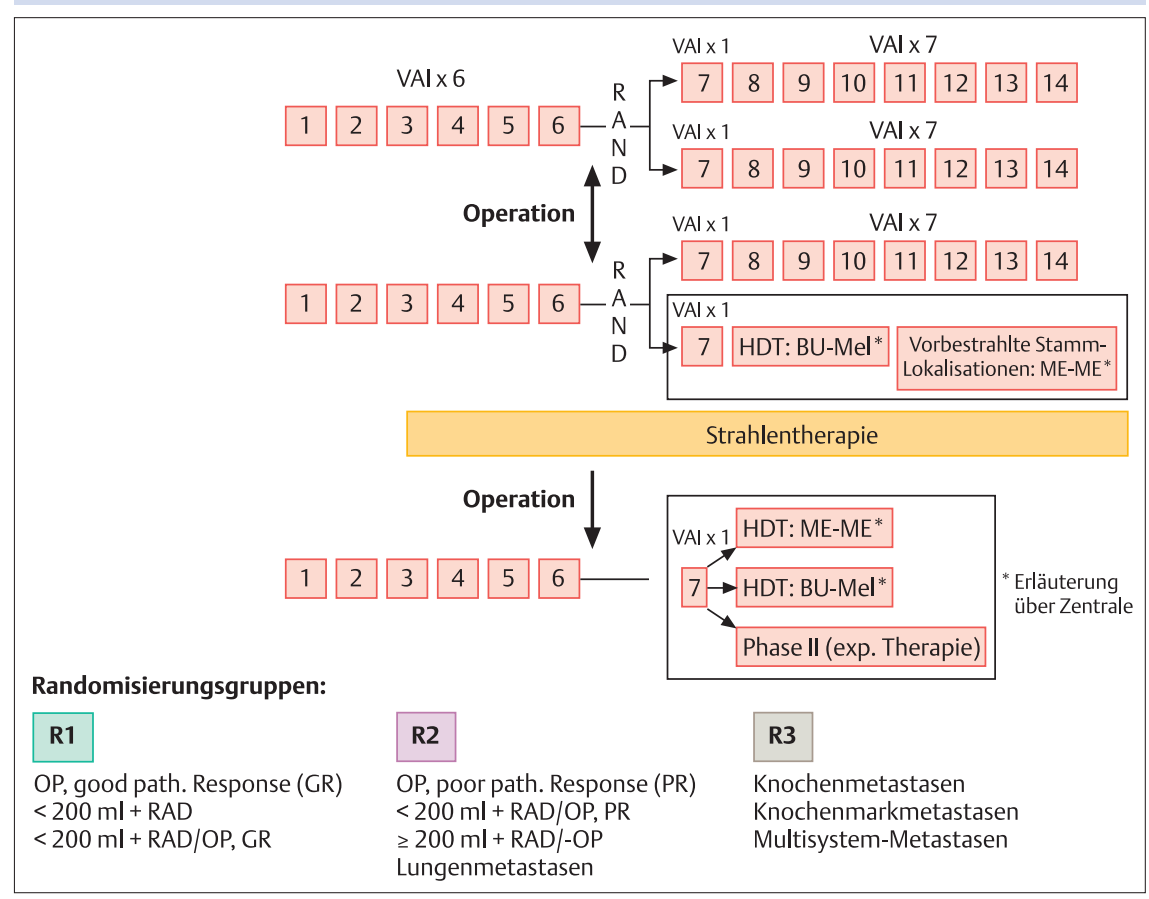

Tab.8 Pathologische Frakturen: Wichtige Punkte in der Behandlung von malignen Knochentumoren mit pathologischer Frakturierung

1 Pathologische Frakturen können durch eine ungenügende Biopsietechnik (Abb. 8,9) entstehen. Zur Frakturvermeidung bei drohender Fraktur sollte möglichst nur tumorbefallenes Weichteilgewebe biopsiert werden. Technisch lässt sich Knochengewebe schonender durch die Anlage kleiner, runder Löcher mit einer Stanzzylinderfräse gewinnen, die anschließend mit Knochenzement verschlossen werden. Ebenso sollte unbedingt eine postoperative Ruhigstellung und Entlastung erfolgen.

2 Im Falle der pathologischen Fraktur muss die interdisziplinär notwendige Therapie in ausgewiesenen Zentren, möglichst unter Studienbedingungen erfolgen.

3 Bei niedrigmalignen Knochentumoren ohne multimodale Therapie kann die kompartmentgerechte Resektion früh erfolgen, falls alle hämatomgefüllten Areale sorgfältig mitentfernt werden. Bei hochmalignen Osteosarkomen kann dies im Rahmen der EURO B.O.S.S.-Studie ebenso primär erfolgen.

Für eine weite Resektion muss im Fall der pathologischen Fraktur wegen Frakturhämatomen immer mehr Gewebeentfernung gefordert werden, als im nicht frakturierten Zustand. Durch lokale Tumoraussaat wird die Prognose bei onkologisch nicht gerechter Teilresektion deutlich verschlechtert und zwingt signifikant häufiger zur sekundären Amputation.

4 Bei pathologischen Frakturen von hochmalignen Knochentumoren muss zunächst die Extremität ggf. mit einem Fixateur extern oder einer Schiene ruhig gestellt werden, um die Chemotherapie im Rahmen des multimodalen Studienprotokolls einzuleiten. Oftmals wir dann durch eine chemoinduzierte Tumorverkleinerung („Downstaging“) auch die Resektion eines frakturierten Bereichs erleichtert.

5 Auch die primäre Amputation muss bei erheblicher Frakturdislokation mit ausgedehnten Hämatomen in ungünstiger Lokalisation mit in die therapeutische Überlegung einbezogen werden, denn die Lokalrezidivrate steigt bei Extremitätenerhalt in diesem Fall von etwa 8 auf $30 \%$. 
Tab. 9 und 10 Wichtige Punkte in der Behandlung von malignen Knochentumoren mit pathologischer Frakturierung

Tab.9 L.B., geb. 1984

Schmerzen OS li seit 2/00

13.3.2000: Biopsie

Diagnose: Osteosarkom Stadium IIB (Abb. 1-4)

Neoadjuvante Chemo n. COSS-96: 3-7/00

5.7.2000: weite Resektion, Tumorprothese (Abb. 5-6) (Mutars, Implantcast $\AA$ )

Adjuvante Chemo bis 12/00

Regelmäßige unauffällige Nachsorge, zuletzt 12/05

Tab.10 L.B., geb. 1984

Schmerzen OS re seit 7/02

19.7.2002: Biopsie (Abb. 10)

Diagnose: Ewing-Sarkom Stadium IIB

Pathologische Fraktur und Stabilisation 9/02 (Abb.11, 12)

Neoadjuvante Chemo n. EURO-EWING 99: 9/02-1/03

21.2.2003: weite Resektion, Femurersatz durch Allograft mit UFN und LCP (Synthes $\AA$ ) (Abb. 13, 14)

Adjuvante Chemotherapie

Regelmäßige unauffällige Nachsorge, zuletzt 2/06 (Abb. 15)

zuwägen, die sich jedoch immer an den o.g. Richtlinien orientieren sollten, um auch in einer gegebenenfalls vorliegenden palliativen Situation dem Patienten eine schmerzfreie und komplikationslose verbleibende Überlebenszeit zu ermöglichen.

\section{Literatur}

${ }^{1}$ Bielack S, Kempf-Bielack B, Delling G et al. Prognostic factors in high-grade osteosarcoma of the extremities or trunk. An analysis of 1702 patients treated on neoadjuvant $\mathrm{Co}$ operative Osteosarcoma Study Group protocols. J Clin Oncol., 2002; 20: 776-90

2 Bacci G, Ferrari S, Donati Dea. Neoadjuvant chemotherapy for osteosarcoma of the extremity in patients in the fourth and fifth decade of life. Oncol Rep 1998; 5: 1259-1263

3 Campanacci M. High grade osteosarcomas. In: tumors. ICMeBast, editor. Wien-New York: Springer-Verlag; 1991

${ }^{4}$ Jürgens $\mathrm{H}$, Exner U, Gadner Hea. Multidisciplinary treatment of primary Ewing's sarcoma of bone. A 6-year experience of a European Cooperative Trial. Cancer 1988; 61(1): 23-32
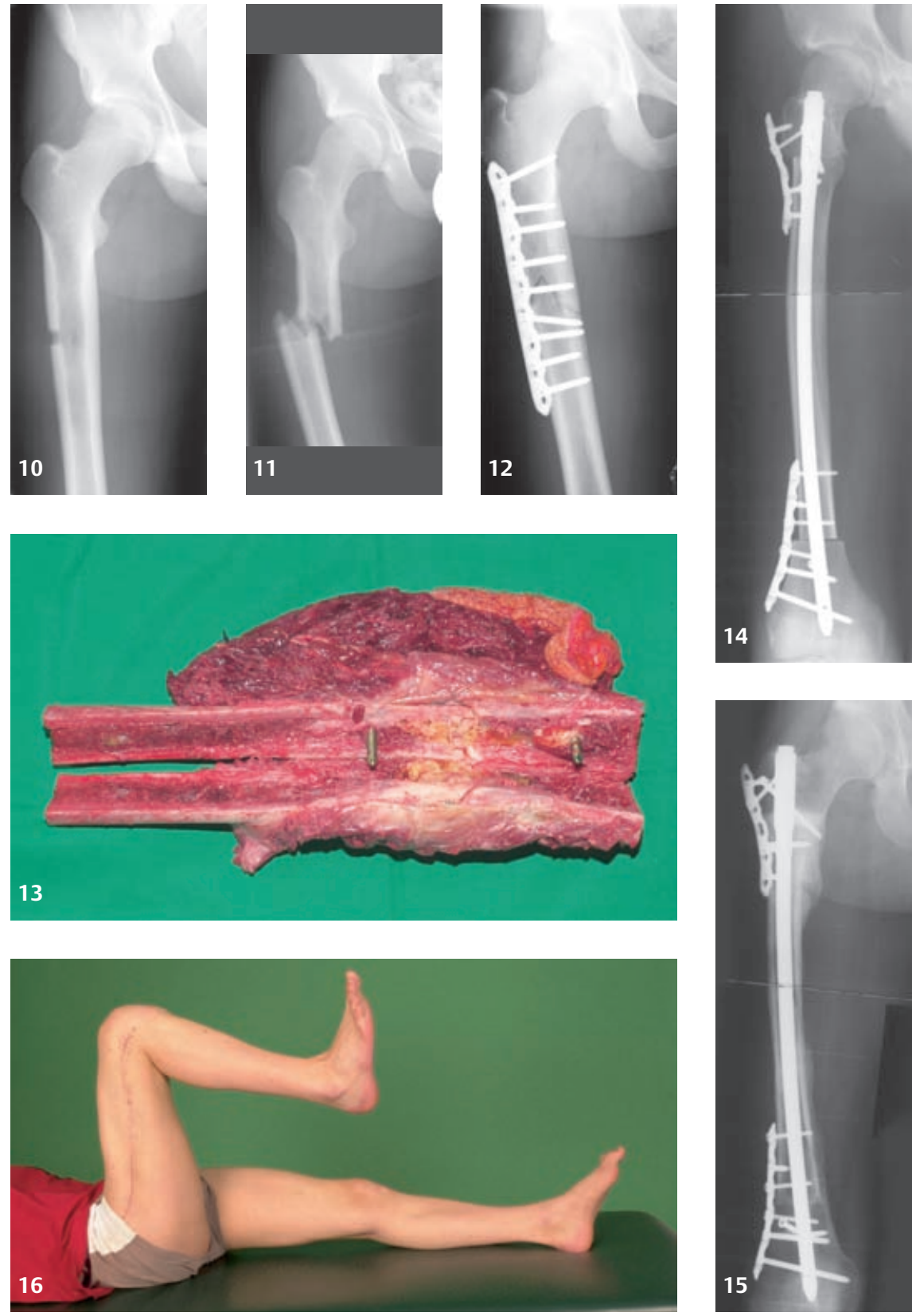

Abb. 10-16 (siehe Tab. 10)

${ }^{5}$ Lane J, Healey J. Diagnosis and management of pathologic fractures. New York: Raven Press; 1993

${ }^{6}$ Malawer M, Link P, Donaldson S. Sarcomas of bone: Philadelphia-New York: LippincottRaven; 1997.

7 Mercuri M, Picci P, Campanacci L, E. R. Dedifferentiated Chondrosarcoma. Skeletal Radiol 1995; 24: 409-416

8 Mirels H. Metastatic disease in long bones. Clin Orthop 1989; 249-256

${ }^{9}$ Mutschler W, Wirbel R. Pathologische Frakturen. Unfallchirurg 1997; 100: 410-429

${ }^{10}$ Salzer-Kuntschik M, Delling G, Beron G, Sigmund R. Morphological grades of regression in osteosarcoma after polychemotherapystudy COSS 80. J Cancer Clin Oncol 1983; 106 (s): 21-24

11 William F, Enneking M. A system of staging musculoskeletal neoplasms. Clin Orthop Rel Res 1986; 204: 9-24
Priv.-Doz. Dr. med. Markus Schultheiss Unfallchirurg

Prof. Dr. med. Lothar Kinzl

Ärztlicher Direktor

Alexandra von Baer

Priv.-Doz. Dr. med. Erich von Hartwig Chefarzt

\section{Dr. med. Markus Arand}

Oberarzt

Universitätsklinikum Ulm

Abteilung für Unfall-, Hand-, Plastische und Wiederherstellungschirurgie

Steinhövelstraße 9

89081 Ulm
Assistenzarzt 